\title{
Costo-efectividad de la vacunación contra influenza pandémica en mujeres embarazadas en Colombia
}

\author{
Luz Angela Chocontá-Piraquive, ${ }^{1}$ Nelson Alvis Guzmán ${ }^{2}$ \\ y Fernando De la Hoz Restrepo'
}

Forma de citar Chocontá-Piraquive LA, Alvis Guzmán N, De la Hoz Restrepo F. Costo-efectividad de la vacunación contra influenza pandémica en mujeres embarazadas en Colombia. Rev Panam Salud Publica. 2012;31(6):447-53.

RESUMEN Objetivo. Estimar la relación costo-efectividad de la vacunación contra influenza pandémica A (H1N1) 2009 en mujeres embarazadas en Colombia durante la segunda onda de la pandemia.

Métodos. Se construyó un árbol de decisiones que simulaba los resultados sanitarios (muertes y años potenciales de vida perdidos, APVP) en dos cohortes de mujeres embarazadas, una vacunada y otra sin vacunar. Los parámetros del modelo fueron extraídos de la literatura científica y los costos se estimaron a partir de un estudio previo. Se calcularon razones de costo-efectividad incrementales (RCEI).

Resultados. La vacunación de embarazadas contra influenza pandémica habría evitado entre 4664 y 15741 consultas ambulatorias y entre 119 y 401 hospitalizaciones. Los costos de atención evitados serían de US\$249530 a US\$ 842 163. Para el escenario base, vacunar embarazadas sería costo-efectivo (RCEI/APVP evitado US\$ 7 657). Esta RCEI fue sensible a la letalidad de la enfermedad; en escenarios de baja letalidad la vacunación no sería costo-efectiva en Colombia.

Conclusiones. La vacunación en embarazadas contra influenza pandémica es costo-efectiva en un escenario de alta mortalidad. La evidencia existente de que las mujeres embarazadas tienen mayor riesgo de presentar complicaciones y de que la vacuna es segura justificaría su uso en embarazadas.

Palabras clave Costos y análisis de costo; subtipo H1N1 del virus de la influenza A; vacunas contra la influenza; mujeres embarazadas; cobertura de vacunación; Colombia.

La primera pandemia de influenza del siglo XXI fue declarada el 11 de junio de 2009 por la Organización Mundial de la Salud (OMS), después del aislamiento de

Universidad Nacional de Colombia, Facultad de Medicina, Grupo de Epidemiología y Evaluación en Salud Pública, Bogotá, D.C., Colombia.

La correspondencia se debe dirigir a Luz Angela Chocontá-Piraquive. Correo electrónico: lachocontap@unal.edu.co

2 Universidad de Cartagena, Facultad de Ciencias Económicas, Grupo de Investigación en Economía de la Salud, Cartagena, Colombia. un nuevo virus de influenza de origen porcino en el sur de California (Estados Unidos) y de un aumento inusitado en la morbi-mortalidad por neumonía en México (1). La vacunación contra el nuevo virus de influenza A (H1N1) empezó a finales de 2009 en países en desarrollo y los estudios iniciales mostraron que la vacuna era segura y que una sola dosis producía una respuesta inmune adecuada (2-8).

Inicialmente se recomendó vacunar a los siguientes grupos: mujeres embaraza- das, contactos cercanos de niños menores de 6 meses, personal de salud, menores de 24 años y personas de cualquier edad con enfermedades crónicas asociadas a complicaciones por influenza estacional (9). Históricamente, las embarazadas han presentado altas tasas de mortalidad y de hospitalización durante las pandemias de influenza y las temporadas de influenza anuales, patrón que se confirmó durante la pandemia del virus A (H1N1) 2009 (10-14). En Australia, el hecho de que las 
mujeres embarazadas tenían cinco a seis veces más riesgo de ser hospitalizadas o ingresar en unidades de cuidados intensivos (UCI) (15) por influenza reforzó la recomendación de administrar la vacuna en ese grupo de población.

El Ministerio de la Protección Social (MPS) de Colombia adquirió dos millones de dosis de vacuna contra influenza A (H1N1) 2009 para la inmunización de personas en alto riesgo de complicaciones durante la segunda onda de la pandemia que se esperaba para el primer semestre de 2010. El presente estudio tuvo justamente como objetivo estimar la relación costo-efectividad de la vacunación contra influenza pandémica A (H1N1) 2009 en mujeres embarazadas de Colombia durante la segunda onda de la pandemia.

\section{MATERIALES Y MÉTODOS}

\section{Modelo}

Se realizó un estudio de evaluación económica completa. Se construyó un árbol de decisiones para evaluar la relación de costo-efectividad de la vacunación contra influenza A (H1N1) en embarazadas colombianas dentro de la estrategia de mitigación del impacto de la segunda onda de la pandemia. Como se observa en la figura 1, las alternativas evaluadas fueron "vacunar" y "no vacunar" a las embarazadas, seguida cada rama del árbol por iguales desenlaces intermedios (infección y atención) y finales (vive o muere). Se asumieron diferentes niveles de cobertura y de efectividad de la vacuna así como un rango de tasas de ataque. Como medida de desenlace se tomaron los casos ambulatorios y hospitalarios, las muertes y los años potencia-

FIGURA 1. Árbol de decisiones para modelar la vacunación contra influenza A (H1N1) 2009 en embarazadas, Colombia, 2009

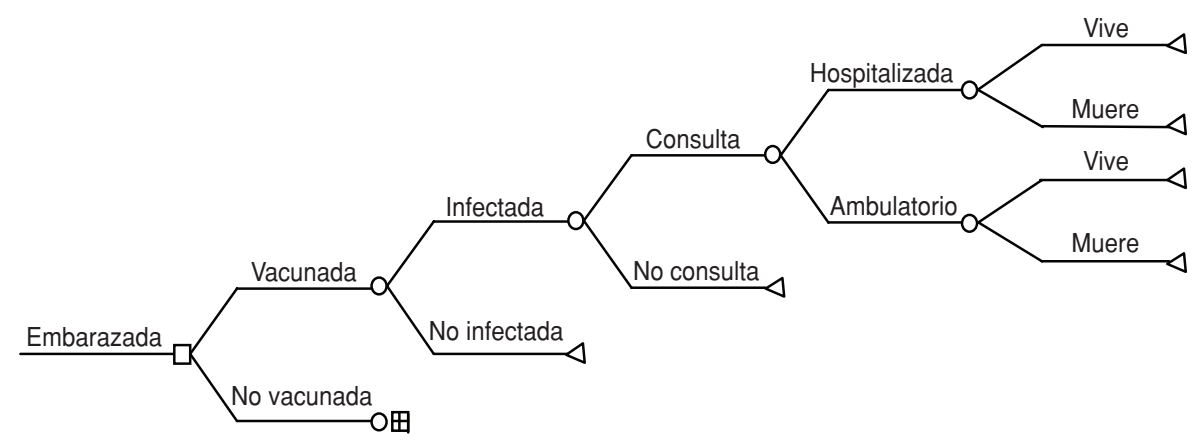

Fuente: elaboración de los autores. les de vida perdidos (APVP) por muerte prematura. Únicamente se consideraron los costos médicos directos.

Se calcularon razones de costo-efectividad incremental (RCEI) definidas como el costo por APVP y muerte evitados. Se asumió que si la RCEI era inferior a tres veces el valor del producto interno bruto (PIB) de Colombia para 2008, la vacunación sería considerada costo-efectiva, mientras que si estaba por debajo de 1 (un) valor del PIB per cápita sería considerada altamente costo-efectiva (16). El horizonte temporal del análisis fue de un año, por lo cual no se utilizaron tasas de descuento. La perspectiva de análisis fue la del sistema de salud.

El modelo se construyó para la segunda onda pandémica. Para la primera onda de la epidemia en Colombia se estimó una tasa de ataque de $10 \%$, que habría generado alrededor de 50357 casos de infección por influenza A (H1N1) 2009 en mujeres embarazadas. Para la segunda onda se asumió que la tasa de ataque disminuyó $1 \%$, dado que todas las mujeres infectadas en la primera onda dejaron de ser susceptibles.

Se realizó una revisión de la literatura para identificar los indicadores epidemiológicos de interés para influenza pandémica: incidencia de influenza pandémica, tasa de ataque, razón caso hospitalizado/caso de infección, razón muerte/caso infectado, razón muerte/ caso que consulta o que se hospitaliza y costos de enfermedad asociada a influenza pandémica. La revisión incluyó búsquedas en PubMed y en revistas como JAMA, The New England Journal of Medicine, Lancet, BMJ y Eurosurveillance, en donde se crearon sitios especializados que recopilaban todas las publicaciones sobre la pandemia. Ade-

\footnotetext{
Fuente: elaboración de los autores.
}

más, fueron consultados los informes de la OMS y de los Centros para el Control y la Prevención de Enfermedades (CDC) de Estados Unidos. Dado que la revisión se hizo en tiempo real (mientras ocurría la pandemia) y los datos eran en su totalidad de estudios observacionales, no se hizo una revisión sistemática ni meta-análisis. Los valores elegidos corresponden a los rangos obtenidos de los estudios disponibles al momento de la construcción del modelo.

Se supuso que el riesgo de infectarse de las mujeres embarazadas es el mismo que el de la población general y el riesgo de complicaciones es el mismo en cualquiera de los tres trimestres del embarazo. También se supuso que todas las mujeres embarazadas con un episodio de enfermedad respiratoria aguda consultaron a un servicio médico, ya fuera atención ambulatoria o urgencias. Para el cálculo de los APVP, se tomó la edad promedio de muerte notificada para los casos de mortalidad materna en Bogotá —que fue de 26 años- y la esperanza de vida para mujeres calculada por el Departamento Administrativo Nacional de Estadísticas (DANE), 77 años (17).

Dado que las embarazadas presentan una respuesta inmune igual a la de la población general, en el modelo solo reciben una dosis de vacuna (18). La prevalencia de embarazo se tomó de la Encuesta Nacional de Demografía y Salud (ENDS) y se aplicó a la población de mujeres de 15 a 49 años de edad para 2008, obtenida de las proyecciones de población del DANE. Los límites inferior y superior de los parámetros para las estimaciones se construyeron con una variación de $20 \%$ en tasa de ataque, infectados sintomáticos, letalidad y número de consultas por caso. Se supuso que una variabilidad de $\pm 20 \%$ para estos parámetros pone a prueba la robustez del modelo, ya que los cambios en los valores absolutos que se simulan se apartan bastante del caso base. En el cuadro 1 se presentan los valores del caso base para cada una de las variables.

\section{Indicadores de influenza}

Durante el curso de la pandemia se informaron diversas estimaciones de tasas de ataque en un rango que iba de 28,5\% (La Gloria, México) en un período de un mes hasta $1 \%$ (Reino Unido) en un período de seis meses (19-22). En Francia se realizó un estudio serológico en 
CUADRO 1. Parámetros epidemiológicos y de costos para el modelo de evaluación de costo-efectividad de la vacunación contra influenza A (H1N1) 2009 en embarazas, Colombia, 2010

\begin{tabular}{lcr}
\hline \multicolumn{1}{c}{ Variable } & Caso base & \multicolumn{1}{c}{ Referencia } \\
\hline Población de embarazadas & 503570 & $(42,43)$ \\
Tasa de ataque & $9 \%$ & $(19,20,22-24)$ \\
Sintomáticos & $66,9 \%$ & $(25)$ \\
Hospitalizaciones & $1 \%$ & $(19,21,22,23)$ \\
Letalidad & $0,05 \%(0,02 \%-0,07 \%)$ & $(19,21)$ \\
Edad de fallecimiento & $\sim 26$ años & $(17)$ \\
Esperanza de vida & 77 años & $(42)$ \\
Costos $(U S \$)^{a}$ & & $(31)$ \\
$\quad$ Consultas & 11 & $(31)$ \\
$\quad$ Hospitalizaciones & 2500 & $(44)$ \\
$\quad$ Vacuna/dosis & 9 & $(26)$ \\
Vacuna & & {$[$ Supuesto] } \\
$\quad$ Efectividad & 60 & \\
$\quad$ Cobertura & 50 &
\end{tabular}

Fuente: elaboración de los autores.

a Tipo de cambio $\$ 2243$ por US\$1,0 de 2008 (32).

mujeres embarazadas a partir del cual se estimó una tasa de ataque cercana a $10 \%$ (23), un valor moderado dentro del rango descrito por lo cual fue usado para el presente modelo. La proporción de sintomáticos hospitalizados se estimó en $1 \%$ y la proporción de casos fatales se supuso en $0,05 \%$ dentro del rango de $0,02 \%$ a $0,07 \%$ según lo descrito en la literatura $(19,21,23,24)$. Debido a que las mujeres embarazadas son un grupo en riesgo de complicaciones por influenza, se supuso que cada mujer embarazada sintomática consultó al menos una vez a su servicio médico.

Existe evidencia limitada de la proporción de sujetos que desarrollan síntomas al infectarse con el virus de influenza estacional y ninguna evidencia para influenza pandémica. De allí que se haya usado el porcentaje de sintomáticos para influenza estacional $(66,9 \%)$ obtenido de un metaanálisis realizado por Carrat y colaboradores (25), en el cual se incluyeron 56 estudios de influenza en voluntarios sanos (1 280 participantes).

\section{Vacuna}

Se supuso un rango de efectividad de $50 \%$ a $70 \%$ para hospitalizaciones y consultas ambulatorias correspondiente a la efectividad para la vacuna contra influenza estacional tomada de un metaanálisis (26). Un informe preliminar de la OMS publicado en abril de 2010 mostró una efectividad mayor a $70 \%$ en 11 estudios realizados en países europeos (27). En el presente modelo se supuso que las mujeres recibían una sola dosis de vacuna $(3,7,28)$ y que se lograba una co- bertura de $40 \%$ a $70 \%$, similar o más alta que la lograda por Estados Unidos (38\%) $(29,30)$, ya que en Colombia se hizo una amplia difusión de las recomendaciones de aplicación de esta vacuna.

\section{Costos y costo-efectividad}

Los costos de las consultas y hospitalizaciones se extrajeron de un estudio de costo-efectividad de la vacuna contra influenza estacional para Colombia, en el cual se realizó un estudio de microcosteo de pacientes con diagnóstico de neumonías, y se revisaron los registros individuales de prestación de servicios de una entidad aseguradora en salud de cobertura nacional (31). El costo total de casos se estimó multiplicando el número de casos por el costo de atención ambulatoria u hospitalaria derivado del estudio citado. El precio de la vacuna por dosis fue de US\$ 8 más 15\% en costos administrativos, que fue el valor pagado por el MPS al fondo rotatorio de la Organización Panamericana de la Salud. El tipo de cambio utilizado — 2243,59 / US\$ 1,0- fue el reportado por el Banco de la República de Colombia al 31 de diciembre de 2008 (32). Se llevó a cabo un análisis de sensibilidad determinístico univariado tipo tornado y se realizó un análisis por escenarios.

\section{RESULTADOS}

\section{Carga de enfermedad}

En el cuadro 2 se muestra la carga de enfermedad en embarazadas durante la segunda onda pandémica. Como se
CUADRO 2. Estimaciones del impacto de la enfermedad por influenza A (H1N1) 2009 en mujeres embarazadas, Colombia, 2010

\begin{tabular}{lcc}
\hline & Segunda onda & Intervalo $^{\text {a }}$ \\
\hline Infectados & 45321 & $36257-54385$ \\
Consultas & 30365 & $15547-52471$ \\
Hospitalizados & 304 & $155-525$ \\
Muertes & 15 & $8-26$ \\
APVP & 774 & $396-1338$ \\
Costos (US\$) & 1093150 & $559693-1888963$ \\
\hline
\end{tabular}

Fuente: elaboración de los autores.

Nota: APVP: años potenciales de vida perdidos.

a Los límites inferior y superior se construyen con una variación sincrónica de (+/-) 20\% para los parámetros: tasa de ataque, proporción de sintomáticos, letalidad, tasa de hospitalización y número de consultas por caso.

b Tipo de cambio \$2 243 por US\$1,0 de 2008 (32).

puede observar, en ausencia de vacunación se producirían alrededor de 30000 consultas y 300 hospitalizaciones con costos directos de atención cercanos a un millón de dólares, y se perderían alrededor de 774 APVP, es decir, 1,54 APVP por cada 1000 mujeres embarazadas. En el cuadro 3 se presenta la carga de enfermedad evitada con la vacunación de embarazadas. En el caso base, con una efectividad de la vacuna de $60 \%$ y una cobertura de $50 \%$ se evitan 0,46 APVP por cada 1000 mujeres. En promedio se ahorrarían US\$ 93525 en costos de atención ambulatoria y US\$ 365598 en costos de hospitalizaciones.

\section{Costo-efectividad}

En el cuadro 4 se pueden ver los resultados del análisis de costo-efectividad en dos escenarios, el caso base y otro más conservador donde se considera baja letalidad $(0,02 \%)$, baja cobertura de vacunación $(40 \%)$ y alta efectividad de la vacuna $(70 \%)$. La RCEI estimada para el caso base fue de US\$ 7567 por APVP evitado, mientras que para el escenario conservador fue de US\$15 610, lo que ya no sería costo-efectivo en Colombia.

\section{Análisis de sensibilidad}

La figura 2 corresponde al gráfico de tornado, donde se observa que la razón de costo-efectividad de la vacunación fue muy sensible a los cambios en la letalidad de la enfermedad, la proporción de infecciones sintomáticas y la tasa de ataque. Al disminuir en $20 \%$ los parámetros de tasa de ataque, proporción de sintomáticos, consultas, hospitalizaciones y muertes, la RCEI estimada sería de US\$ 16955 por APVP evitado, sobrepa- 
CUADRO 3. Estimaciones de consultas, hospitalizaciones, muertes y costos evitados con la vacunación contra influenza $A$ (H1N1) 2009 en mujeres embarazadas, para la segunda ola de la pandemia, Colombia, 2010

\begin{tabular}{lrrr}
\hline & Caso base & \multicolumn{2}{c}{ Intervalo $^{\text {a }}$} \\
\hline Consultas & 9110 & 4664 & 15741 \\
Hospitalizaciones & 155 & 79 & 268 \\
Muertes & 5 & 2 & 8 \\
Años de vida perdidos & 232 & 119 & 401 \\
Costos de atención (US $\$)^{b}$ & 487363 & 249530 & 842163 \\
\hline Fuente: elaboración de los autores. \\
a Los límites inferior y superior se construyen con una variación sincrónica de (+/-) \\
20\% en los parámetros: tasa de ataque, sintomáticos, letalidad, tasa de hospitali- \\
zación y consulta por caso. \\
b Tipo de cambio $\$ 2$ 243 por US $\$ 1,0$ de 2008 (32).
\end{tabular}

CUADRO 4. Índice de costo-efectividad de la vacunación contra influenza A (H1N1) 2009 por APVP y muerte evitados en diferentes escenarios, Colombia, 2010

\begin{tabular}{lrrr}
\hline & Caso base & \multicolumn{2}{c}{ Intervalo } \\
\hline Escenario 1 & & & \\
Costo por APVP evitado (US\$) & 7657 & 16955 & 3547 \\
$\quad$ Costo por muerte evitada (US\$) & 390512 & 864704 & 180912 \\
Escenario 2 & & & \\
Costo por APVP evitado (US\$) $^{\mathrm{a}}$ & 15610 & - & - \\
Costo por muerte evitada (US\$) $^{\mathrm{b}}$ & 796098 & \multicolumn{1}{c}{-} & \multicolumn{1}{c}{-} \\
\hline
\end{tabular}

Fuente: elaboración de los autores.

Nota: APVP: años potenciales de vida perdidos;las celdas sin cifras se deben a que el escenario 2 es una variación en los parámetros del escenario 1 y no se hace estimación de intervalos.

a Tipo de cambio $\$ 2243$ por US\$1,0 de 2008 (32).

b El escenario 2 contempla una baja letalidad de la enfermedad $(0,02 \%)$, una baja cobertura de vacunación en embarazadas (40\%) y una alta efectividad de la vacuna $(70 \%)$

FIGURA 2. Diagrama de tornado del análisis de sensibilidad de la relación costo-efectividad de la vacunación de mujeres embarazadas contra influenza A (H1N1) 2009, Colombia, 2009

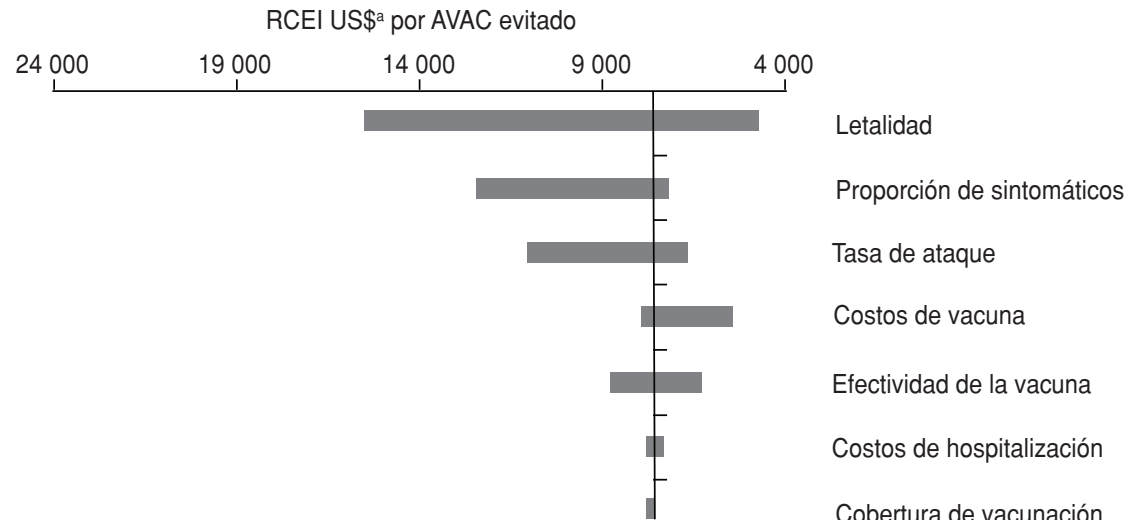

Fuente: elaboración de los autores.

Nota: RCEl: razones de costo-efectividad incremental; AVAC: año de vida ajustado por calidad.

a Tipo de cambio $\$ 2243$ por US\$1,0 de 2008 (32).

sando el límite de costo-efectividad para Colombia (US\$ 14700$)$.

\section{DISCUSIÓN}

Los resultados del presente trabajo revelan que la vacunación de embaraza- das contra el virus pandémico es costoefectiva o muy costo-efectiva en escenarios de alta letalidad de la enfermedad $(\geq 0,05 \%)$. En un escenario con baja letalidad $(\leq 0,02 \%)$ y bajas coberturas de vacunación $(40 \%)$, la RCEI sobrepasaría el límite de la disponibilidad a pagar de US\$ 14700 . Estos datos ilustran la importancia que tiene la consideración de la incertidumbre acerca del comportamiento epidemiológico de la infección por influenza pandémica al momento de evaluar el uso de una vacuna.

Estudios similares de evaluación económica de la vacunación contra el virus de influenza A (H1N1) 2009 resultaron favorables a la vacuna (33-36). Sin embargo, en los primeros estudios se supusieron altas tasas de ataque, así como importantes proporciones de hospitalizaciones y de letalidad, altos costos de la vacuna y la necesidad de aplicar dos dosis. En la mayoría de estudios también se supuso que la vacunación ocurriría antes o durante las primeras semanas de la epidemia, momento en el cual las estrategias de vacunación son muy costo-efectivas.

Una evaluación económica de la vacuna llevada a cabo por Baguelin y colaboradores (37) mostró que ante la segunda onda epidémica en Inglaterra, solo la vacunación de los grupos de riesgo sería costo-efectiva bajo un umbral de costo-efectividad de $£ 20000$ (US\$ 30 000) por año de vida ajustado por calidad (AVAC) ganado. Los grupos de riesgo correspondían a la población susceptible de enfermedad severa o muerte por influenza estacional, incluyendo a las mujeres embarazadas. La extensión de la vacunación a los grupos de bajo riesgo habría ahorrado muy pocas muertes, pero evitaría un importante número de casos, especialmente en niños.

Al momento de realizarse el presente estudio, solo se había publicado un artículo de costo-efectividad en embarazadas (38). Dicho trabajo, llevado a cabo por investigadores de la Universidad de Pittsburg en Pensilvania (Estados Unidos), evaluó la inmunización con una o dos dosis de vacuna contra enfermedad por influenza confirmada por laboratorio y concluyó que la vacunación era costo-efectiva suponiendo tasas de ataque mayores de 7,5\%, letalidad mayor de $1,05 \%$ y eficacia de la vacuna de 70\% (umbral RCEI US\$ 50 000/AVAC ganado). Este modelo tuvo en cuenta el desenlace de la enfermedad para la mujer, las consecuencias para el feto y las reacciones adversas a la vacunación para ambos. En ese estudio, para una tasa de ataque de $10 \%$ como la supuesta en el presente trabajo, la RCEI por AVAC ganado sería de US\$9165 en el escenario de mortalidad del $1 \%$. 
Cabe señalar que no se tuvieron en cuenta los posibles efectos adversos de la vacunación, porque no hay evidencia de que las mujeres embarazadas o los productos entrañen un riesgo elevado de efectos adversos al recibir la vacuna estacional. De acuerdo con el sistema de informe de eventos adversos de vacunaciones (VAERS, por sus siglas en inglés) en Estados Unidos en el período de 2000 a 2003 se registraron 26 eventos en los 42 días posteriores a la aplicación de la vacuna -entre ellos tres abortos-entre 2 millones de mujeres embarazadas vacunadas.

Para la pandemia de influenza A (H1N1) 2009, a mayo de 2010 se registraron 11180 informes de eventos adversos, de los cuales $92 \%$ se clasificaron como leves y solo $8 \%$ como severos, aunque sin un patrón de presentación que indique asociación con la vacuna. Los casos de síndrome de Guillain-Barré se han presentado con una frecuencia igual a la de años anteriores $(26,39)$. Proyectar estos indicadores a la población aquí estudiada arrojaría como resultado que se presentarían de 7 a 10 eventos adversos potencialmente asociados a la vacunación.

Todas las estimaciones sobre la pandemia están sujetas a una importante variabilidad generada por la incertidumbre en la extensión de la infección. En los estudios descritos, incluido el presente, la tasa de ataque al final de la pandemia y la letalidad son las dos variables principales de las que depende el grado de costo-efectividad de la vacuna. Estudios publicados al final de la primera onda, con estimaciones basadas en sistemas de vigilancia epidemiológica, reportaron tasas de ataque de casos sintomáticos bastante menores a las proyectadas al inicio de la pandemia, y no se sabe mucho acerca de la extensión de la infección con sintomatología leve o totalmente asintomática.

Un estudio seroepidemiológico realizado en el Reino Unido mostró niveles de infección mucho más altos que los estimados por la vigilancia clínica. Informa que, en las regiones con alta incidencia, uno de cada tres niños resultó infectado con el virus de influenza pandémica durante la primera onda de la pandemia y -además- población con niveles de anticuerpos protectores previos a la aparición del nuevo virus que oscilan entre $1,8 \%$ en los menores de 5 años a $23 \%$ en los mayores de 65 . Esto podría indicar una alta proporción de casos asintomáticos, lo cual tiene repercusión en las evaluaciones de costo-efectividad de la vacuna. El estudio de costo-efectividad de Baguelin tiene en cuenta estos hallazgos y asume un porcentaje de sintomáticos de $37 \%$ (37).

Las diferencias regionales en la distribución de la infección también fueron notables. En el estudio serológico del Reino Unido la incidencia de infección fue mucho más alta para Londres y Midland del occidente que para las otras regiones. Mientras que en Londres los niveles de anticuerpos en menores de 25 años de edad aumentaron más de $20 \%$, en las otras regiones en menores de 15 años lo hicieron apenas 6\%. Estos resultados también sugieren el papel que juegan los menores en la transmisión de la infección: la vacunación de niños escolarizados al inicio de la pandemia podría haber sido una estrategia efectiva para mitigar las tasas de ataque poblacional (40).

En relación con la efectividad de la vacuna, un informe del Centro Europeo para la Prevención y Control de Enfermedades (CEPCE) describe los hallazgos preliminares de varios estudios de casos y controles, señalando en todos una efectividad superior a $70 \%$ (41). Estas estimaciones de efectividad presentan amplios intervalos de confianza, probablemente debido al bajo número de sujetos vacunados. Los investigadores consideran que esta efectividad se relaciona consistentemente con los estudios de inmunogenicidad y que podría atribuirse a una alta especificidad de la vacuna contra el virus.

La aceptabilidad de la vacuna contra influenza pandémica también constituye un reto para las autoridades de salud y puede afectar la costo-efectividad de los programas de inmunización. Incluso cuando numerosos estudios han probado la seguridad de la vacuna contra influenza estacional, la vacunación durante el embarazo genera preocupación en las madres por las posibles consecuencias que pueda tener para el embarazo (29). Por otro lado, si bien en el contexto de una pandemia la vacunación puede percibirse como un riesgo mayor dada la falta de estudios de seguridad, la cobertura de inmunización en Estados Unidos fue mayor que la de influenza estacional (30). En Colombia, el Programa Ampliado de Inmunizaciones (PAI) ha logrado coberturas de más de $60 \%$ en menores de 2 años y mayores de 60 años con vacuna estacional de influenza.

Un comentario aparte merece el uso del valor del PIB reportado por el Banco Mundial para Colombia. En menos de 10 años el PIB per cápita de Colombia pasó de US\$ 1900 en el año 2000 a US\$ 4900 en 2008. Tal crecimiento — catalogado como una especie de milagro del desarrollo- ha recibido cuestionamientos de economistas independientes, quienes señalan que más de $70 \%$ se debe a los cambios en la metodología de medición de los últimos años. Aunque el DANE es claro en su justificación del cambio de metodología, no está del todo garantizada la equivalencia en las bases de cálculo que permitan establecer razones entre valores del PIB per cápita de los años 2000 y 2005. Se ha postulado que si se usaran los procedimientos del año 2000, el PIB para Colombia rondaría en realidad US\$ 2 400. En ese escenario, la vacunación no sería costo-efectiva bajo ninguna de las asunciones.

Estos resultados deben interpretarse teniendo en cuenta algunas limitaciones metodológicas. La principal es la dificultad para obtener estimadores del comportamiento epidemiológico de la pandemia, aun cuando se utilizaron las estimaciones más conservadoras publicadas y, en el caso de la proporción de sintomáticos, se hizo uso de la mejor evidencia disponible para influenza estacional. En cuanto a los costos de la enfermedad aplicados, en la literatura revisada no se encontraron estudios previos en países de bajos o medianos ingresos que revelen costos de influenza pandémica en embarazadas, mientras que los reportados en países desarrollados no son comparables, pues los costos de atención son mucho más altos que los estimados para Colombia.

\section{Conclusiones y recomendaciones}

Este estudio muestra que la vacunación de mujeres embarazadas en el contexto de una pandemia de influenza podría ser costo-efectiva, siempre y cuando las autoridades aseguren la disponibilidad oportuna de la vacuna, la aceptabilidad de la población a vacunarse sea alta y el monitoreo de la enfermedad permita evaluaciones en tiempo real. La vacunación contra el virus A (H1N1) 2009 durante la pasada pandemia fue controversial debido a lo poco que se conocía 
acerca de la dinámica de la infección al inicio de la primera onda pandémica, de la efectividad de la vacuna y de su seguridad. No obstante, dada la alta sensibilidad social del tema y la presión por proteger a la población con todos los medios disponibles, es probable que la vacunación de grupos de riesgo haya estado justificada. La aplicación de una vacuna como la desarrollada para la pandemia de influenza implica que los organismos responsables de la salud de la población, incluyendo los aseguradores y las secretarías de salud, tomen medidas para vigilar fallas de la vacuna y potenciales reacciones adversas. El conjunto de autoridades y aseguradores en salud deben aprovechar la experiencia para mejorar sus sistemas de vigilancia, tanto sobre la enfermedad como sobre los eventos adversos relacionados con la vacunación. Además, debe evaluar el uso de estudios serológicos para determinar la extensión de la infección por este tipo de virus.

Financiación: La presente investigación fue financiada por el Ministerio de la Protección Social de Colombia.

\section{REFERENCIAS}

1. Dawood FS, Jain S, Finelli L, Shaw MW, Lindstrom S, Garten RJ, et al. Emergence of a novel swine-origin influenza A (H1N1) virus in humans. N Engl J Med. 2009;360(25): 2605-15.

2. Clark TW, Pareek M, Hoschler K, Dillon $\mathrm{H}$, Nicholson KG, Groth N, et al. Trial of 2009 influenza A (H1N1) monovalent MF59adjuvanted vaccine - Preliminary Report. N Engl J Med. 2009;361(25):2424-35.

3. Greenberg ME, Lai MH, Hartel GF, Wichems $\mathrm{CH}$, Gittleson C, Bennet J, et al. Response to a monovalent 2009 influenza A (H1N1) vaccine. N Engl J Med. 2009;361(25):2405-13.

4. Kelly H, Barr I. Large trials confirm immunogenicity of H1N1 vaccines. Lancet. 2010; 375(9708):6-9.

5. Liang XF, Wang HQ, Wang JZ, Fang HH, Wu J, Zhu FC, et al. Safety and immunogenicity of 2009 pandemic influenza A H1N1 vaccines in China: a multicentre, double-blind, randomised, placebo-controlled trial. Lancet. 2009;375(9708):56-66.

6. Nolan T, McVernon J, Skeljo M, Richmond P, Wadia U, Lambert S, et al. Immunogenicity of a monovalent 2009 influenza A (H1N1) vaccine in infants and children: a randomized trial. JAMA. 2010;303(1):37-46.

7. Plennevaux E, Sheldon E, Blatter M, ReevesHoché MK, Denis M. Immune response after a single vaccination against 2009 influenza A H1N1 in USA: a preliminary report of two randomised controlled phase 2 trials. Lancet. 2010;375(9708):41-8.

8. World Health Organization. Statement from WHO Global Advisory Committee on Vaccine Safety about the safety profile of pandemic influenza A (H1N1) 2009 vaccines. Disponible en: www.who.int/vaccine_safety/ GACVS_H1N1_Vaccine_Safety.pdf Acceso el 6 de marzo de 2012.

9. Centers for Disease Control and Prevention. CDC Advisors Make Recommendations for Use of Vaccine Against Novel H1N1. Disponible en: http://www.cdc.gov/media/pressrel/2009/r090729b.htm Acceso el 6 de marzo de 2012.

10. Boon HL, Tahir AM. Pandemic H1N1 2009 (swine flu) and pregnancy. Elsevier. 2010;20(4):101-6.

11. Rasmussen SA, Jamieson DJ, Bresee JS. Pandemic influenza and pregnant women. Emerg Infect Dis. 2008;14(1):95-100.
12. Jamieson DJ, Honein MA, Rasmussen SA, Williams JL, Swerdlow DL, Biggerstaff MS, et al. H1N1 2009 influenza virus infection during pregnancy in the USA. Lancet. 2009:374(9688):451-8.

13. Siston AM, Rasmussen SA, Honein MA, Fry AM, Seib K, Callaghan WM, et al. Pandemic 2009 influenza A (H1N1) virus illness among pregnant women in the United States. JAMA. 2010;303(15):1517-25.

14. Louie JK, Acosta M, Jamieson DJ, Honein MA, California Pandemic (H1N1) Working Group. Severe 2009 H1N1 influenza in pregnant and postpartum women in California. N Engl J Med. 2010;362(1):27-35.

15. Kelly H, Mercer G, Cheng A. Quantifying the risk of pandemic influenza in pregnancy and indigenous people in Australia in 2009. Euro Surveill. 2009;14(50).

16. World Health Organization. Commission on Macroeconomics and Health. Macroeconomics and health: investing in health for economic development. Report of the Commission on Macroeconomics and Health. Geneve, Switzerland: WHO; 2001.

17. Rey G. El virus pandémico A/H1N1 2009 en Colombia. Seminario enfermedades infecciosas y modelos matemáticos aplicados a la influenza pandémica. Bogotá D.C., Colombia: Universidad Nacional de Colombia; 2009.

18. Mak TK, Mangtani P, Leese J, Watson JM, Pfeifer D. Influenza vaccination in pregnancy: current evidence and selected national policies. Lancet Infect Dis. 2008;8(1):44-52.

19. Donaldson LJ, Rutter PD, Ellis BM, Greaves FE, Mytton OT, Pebody RG, et al. Mortality from pandemic A/H1N1 2009 influenza in England: public health surveillance study. BMJ. 2009;339:5213.

20. Fraser C, Donnelly CA, Cauchemez S, Hanage WP, Van Kerkhove MD, Hollingsworth TD, et al. Pandemic potential of a strain of influenza A (H1N1): early findings. Science. 2009;324(5934):1557-61.

21. Dawood FS, Hope KG, Durrheim DN, Givney R, Fry AM, Dalton CB. Estimating the disease burden of pandemic (H1N1) 2009 virus infection in Hunter New England, Northern New South Wales, Australia, 2009. PLoS One. 2010;5(3):9880.

22. Baker MG, Wilson N, Huang QS, Paine $S$, López L, Bandaranayake D, et al. Pandemic influenza $\mathrm{A}(\mathrm{H} 1 \mathrm{~N} 1) \mathrm{v}$ in New Zealand: the experience from April to August 2009. Euro Surveill. 2009;14(34).

23. Institut de Veille Sanitaire. Bulletin Grippe A(H1N1) 2009 No. 79. Point de situation au 12 janvier 2010. París, Francia: INVS; 2010.

24. Presanis AM, De Angelis D, Hagy A, Reed C, Riley S, Cooper BS, et al. The severity of pandemic H1N1 influenza in the United States, from April to July 2009: a Bayesian analysis. PLoS Med. 2009;6(12):e1000207.

25. Carrat F, Vergu E, Ferguson NM, Lemaitre $\mathrm{M}$, Cauchemez S, Leach S, et al. Time lines of infection and disease in human influenza: a review of volunteer challenge studies. Am J Epidemiol. 2008;167(7):775-85.

26. Moreno J, De la Hoz F, Rico A, Cotes K, Porras A. Efectividad de la vacuna contra influenza: meta-análisis de literatura. Biomédica. 2009; 29(1):87-97.

27. Wood D J. Update on H1N1 vaccine immunogenicity, safety and effectiveness. World Health Organization. 2010. Disponible en: http://www.who.int/ immunization/sage/Influenza_2_H1N1_ vaccine_immunogenicity_D_Wood_SAGE April_2010.pdf Acceso el 7 de marzo de 2012.

28. Liang XF, Wang HQ, Wang JZ, Fang HH, Wu J, Zhu FC, et al. Safety and immunogenicity of 2009 pandemic influenza A H1N1 vaccines in China: a multicentre, double-blind, randomised, placebo-controlled trial. Lancet. 2010;375(9708):56-66.

29. Naleway AL, Smith WJ, Mullooly JP. Delivering influenza vaccine to pregnant women. Epidemiol Rev. 2006;28(1):47-53.

30. Center for Disease Control and Prevention. Interim results: influenza A (H1N1) 2009 monovalent vaccination coverage - United States, October-December 2009. MMWR Morb Mortal Wkly Rep. 2010;59(2):44-8.

31. Porras-Ramírez A, Alvis-Guzmán N, RicoMendoza A, Alvis-Estrada L, CastañedaOrjuela CA, Velandia-González MP, et al. Costo efectividad de la vacunación contra influenza en menores de 2 años y mayores de 65 años en Colombia. Rev Salud Publica. 2009;11(5):689-99.

32. Banco de la República de Colombia. Series estadísticas, tasas de cambio. Disponible en: http://banrep.gov.co/series-estadisticas/ see_ts_cam.htm Acceso el 7 de marzo de 2012. 
33. Khazeni N, Hutton DW, Garber AM, Hupert N, Owens DK. Effectiveness and CostEffectiveness of Vaccination Against Pandemic Influenza (H1N1) 2009. Ann Intern Med. 2012;156(5)

34. Brouwers L, Cakici B, Camitz M, Tegnell A, Boman M. Economic consequences to society of pandemic H1N1 influenza 2009 - preliminary results for Sweden. Euro Surveill. 2009;14(37).

35. Yang Y, Sugimoto JD, Halloran ME, Basta NE, Chao DL, Matrajt L, et al. The transmissibility and control of pandemic influenza A (H1N1) virus. Science. 2009;326(5953):729-33.

36. Sypsa V, Pavlopoulou I, Hatzakis A. Use of an inactivated vaccine in mitigating pandemic influenza $\mathrm{A}(\mathrm{H} 1 \mathrm{~N} 1)$ spread: a modelling study to assess the impact of vaccination timing and prioritisation strategies. Euro Surveill. 2009;14(41):19356.

37. Baguelin $M$, Hoek AJ, Jit M, Flasche S, White PJ, Edmunds WJ. Vaccination against pandemic influenza A/H1N1v in England: a real-time economic evaluation. Vaccine. 2010;28(12):2370-84.

38. Beigi RH, Wiringa AE, Bailey RR, Assi TM, Lee BY. Economic value of seasonal and pandemic influenza vaccination during pregnancy. Clin Infect Dis. 2009;49(12):1784-92.

39. Tamma PD, Ault KA, del Rio C, Steinhoff MC, Halsey NA, Omer SB. Safety of influenza vaccination during pregnancy. Am J Obstet Gynecol. 2009;201(6):547-52.

40. Basta NE, Chao DL, Halloran ME, Matrajt L, Longini Jr IM. Strategies for pandemic and seasonal influenza vaccination of schoolchil- dren in the United States. Am J Epidemiol. 2009;170(6):679-86.

41. Ciancio B, Sprenger M. Pandemic H1N1 Vaccine Effectiveness in Europe: Results of the I-MOVE Studies. Disponible en: http:// www.who.int/vaccine research/diseases/ influenza/feb_17_2011_Bruno_Ciancio_ws. pdf Acceso el 4 de abril de 2012

Manuscrito recibido el 11 de abril de 2011. Aceptado para publicación, tras revisión, el 20 de enero de 2012.

ABSTRACT Objective. Estimating the cost-effectiveness ratio of vaccinating pregnant women against the 2009 pandemic influenza A (H1N1) in Colombia during the second wave of the pandemic.

Cost-effectiveness of vaccinating pregnant women against pandemic influenza in Colombia

Methods. A decision tree was constructed, which simulated the health results (deaths and years of potential life lost [YPLL]) in two cohorts of pregnant women; one vaccinated, the other unvaccinated. The model's parameters were drawn from scientific literature and costs were estimated on the basis of a previous study. Incremental cost-effectiveness ratios (ICER) were calculated.

Results. Vaccinating pregnant women against pandemic flu would have prevented between 4664 and 15741 outpatient visits and between 119 and 401 hospitalizations. Between US\$ 249530 and US\$ 842163 in costs of care would have been avoided. For the base scenario, vaccinating pregnant women would be cost-effective (ICER/YPLL avoided US\$ 7 657). This ICER was responsive to the disease's fatality rate; vaccination would not be cost-effective in Colombia in scenarios with a lower fatality rate.

Conclusions. Vaccinating pregnant women against pandemic influenza is costeffective in a scenario with a high mortality rate. The existing evidence that pregnant women are at increased risk of complications and that the vaccine is safe would justify its use in pregnant women.

Key words Cost and cost analysis; influenza A virus, H1N1 subtype; influenza vaccines; pregnant women; immunization coverage; Colombia. 